\title{
Structural and Electronic Properties of the BN, BP and BAs in the Different Phases of Zinc-Blende, $\mathrm{NaCl}$ and $\mathrm{CsCl}$
}

\author{
K. BencheriF ${ }^{a, b, *}$, A. YAkoubi ${ }^{a}$ And H. Mebtouche ${ }^{a}$ \\ ${ }^{a}$ Laboratory of Study of Materials and Instrumentations Optic (LEMIO), Physics Department, \\ University of Djilali Liabes, Sidi Bel Abbes, 22000, Algeria \\ ${ }^{b}$ Center University Belhadj Bouchaib of Ain Témouchent, 46000, Algeria \\ (Received November 26, 2016; in final form January 17, 2017)
}

\begin{abstract}
In this work we used the first $a b$ initio calculations to study the stability of the binary alloys BN, BP and BAs and their behavior in the different phases of zinc-blende, Nacl and $\mathrm{CsCl}$. The full potential linearized augmented plane wave method was employed within density functional theory. Our results show the difference in the calculated structural properties and the band structure is obtained for the zinc-blende structure. We have investigated the lattice parameters and band gap energies. We also give the valence charge density at a high pressure and the analysis of the density of states.
\end{abstract}

DOI: 10.12693/APhysPolA.131.209

PACS/topics: binary alloys, ab initio calculations, lattice parameter, high pressure, band-structure, charge density

\section{Introduction}

The boron-based compounds belong to the family of III-V semiconductors. These are compounds with a large gap, particularly interesting for their applications in the field of high temperatures and for the realization of opto-electronic components. The small core size of the boron atom $\mathrm{B}$ and the absence of $p$ electrons explain their properties. Boron has a deep and localized pseudo potential compared to other atoms in the same column of the periodic table. The preliminary calculations of the ionicity $[1,2]$, illustrate these features. The compounds $\mathrm{BP}, \mathrm{BAs}$ and $\mathrm{BSb}$ crystallize, meanwhile, in the zinc blende structure although there are many theoretical first-principle [3, 4] devoted to structural and electronic properties of $\mathrm{BN}, \mathrm{BP}$ and $\mathrm{BAs}$ in their cubic phase, there are very few experimental data on these compounds $[5,6]$. The fundamental properties (not the network to balance and rigidity modulus) and electronic properties of $\mathrm{BN}, \mathrm{BP}$ and BAs were studied by Wentzcovitch et al. $[7,8]$ which also discussed the properties of these materials to high pressure [9].

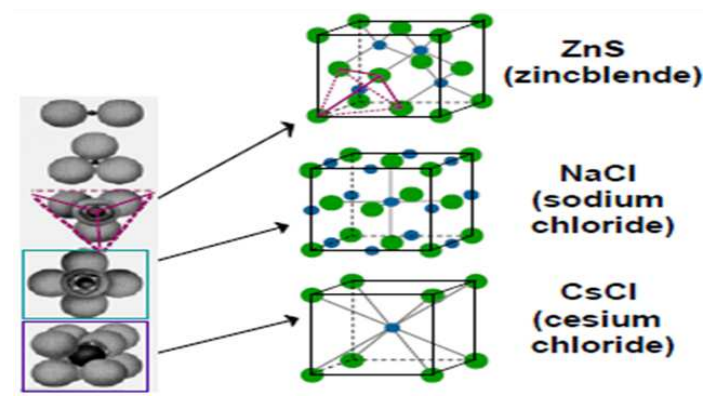

*corresponding author; e-mail: bencherifeim@gmail.com

\section{Computational method}

All of these studies are based on the local density approximation (LDA) associated with the density of functional theory (DFT). The self-energy is usually approximated using a perturbative expansion with respect to the quasi-particle interaction. The quantitative calculation of excitation energies in the binary alloys is the approximation GWA. In this approximation, the selfenergy is the product of the single-particle Green function $\mathrm{G}$ and the screened interaction $\mathrm{W}$. Hedin first proposed the GWA for the computation of quasiparticle energies in 1965. However, the approach was not applied to large-scale, numerical electronic structure calculations. The resulting band gaps compare much better with experiment. To provide estimates of cohesive energies of these compounds, Surh et al. [10] used the GW approximation to consider the effects of exchange and correlation of quasiparticles and recently, Rubio [11] made a calculation of the cubic phase of $\mathrm{BN}$, we note the work of Ferhat et al. [12] and as well as Zaoui et al. [13]. We use the full potential linearized augmented plane wave (FP-LAPW) method implemented in the WIEN97 program [14] to study BN, BP and BAs in the zinc blende phase, $\mathrm{NaCl}$ (rocksalt) and $\mathrm{CsCl}$. All of these studies is based on the LDA [15] associated with the theory of DFT $[16,17]$.

\section{Results and discussion}

Variation of the total energy versus atomic volume of $\mathrm{BN}, \mathrm{BP}$, and $\mathrm{BAs}$ for the different phases $\mathrm{ZB}, \mathrm{NaCl}$, and $\mathrm{CsCl}$ are shown in Figs. 1, 2, and 3 and it is obvious from these curves that the $\mathrm{ZB}$ structure is the most stable at ambient pressure, which is consistent with the experimental results. The corresponding equilibrium lattice parameter, bulk modulus and its pressure derivative are given in Tables I, II, and III for each compound for ZB, 
$\mathrm{NaCl}$ and $\mathrm{CsCL}$ structures. We see, from this table, that our calculated values are in better agreement with experiment than the other calculations. We confirm this result with the only existing one, which was recently performed by Zaoui and El Haj Hassan [18] using LDA within DFT and the pseudo potential method.

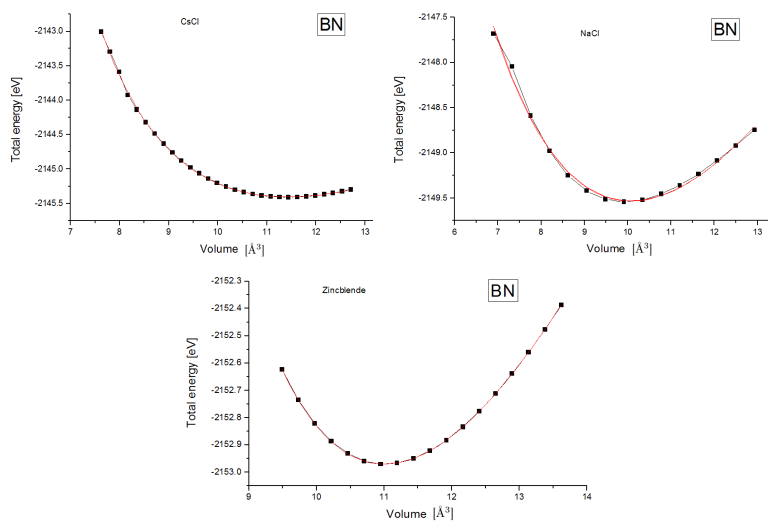

Fig. 1. Variation of the total energy versus volume atomic of $\mathrm{BN}$ for zinc blende, $\mathrm{NaCl}$, and $\mathrm{CsCl}$.

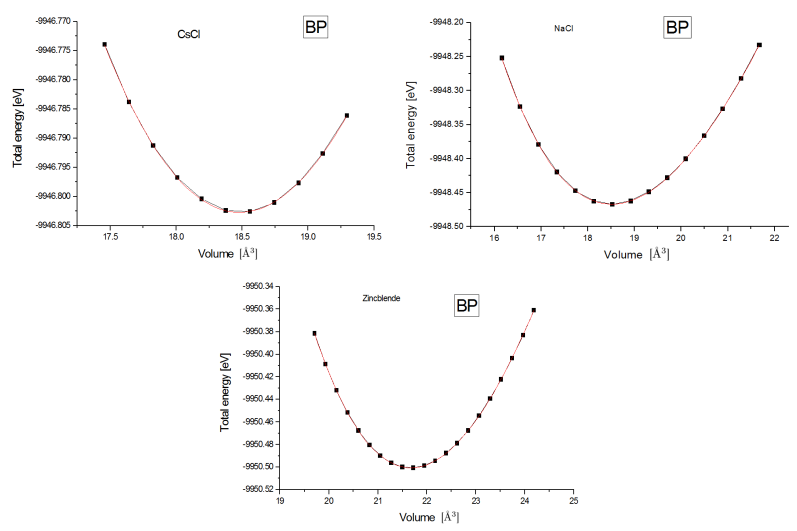

Fig. 2. Variation of the total energy versus volume atomic of $\mathrm{BP}$ for zinc blende, $\mathrm{NaCl}$, and $\mathrm{CsCl}$.

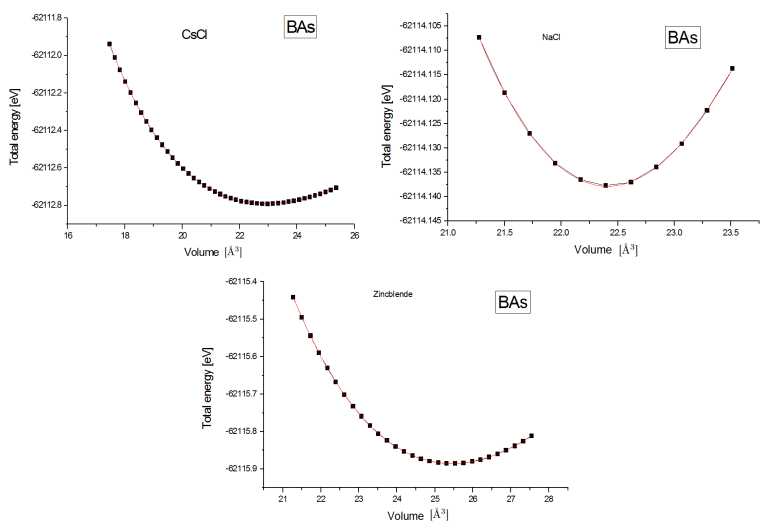

Fig. 3. Variation of the total energy versus volume atomic of BAs for zinc blende, $\mathrm{NaCl}$, and $\mathrm{CsCl}$.
TABLE I

Structural properties of $\mathrm{BN}$ for zinc blende, $\mathrm{NaCl}$, and $\mathrm{CsCl}$.

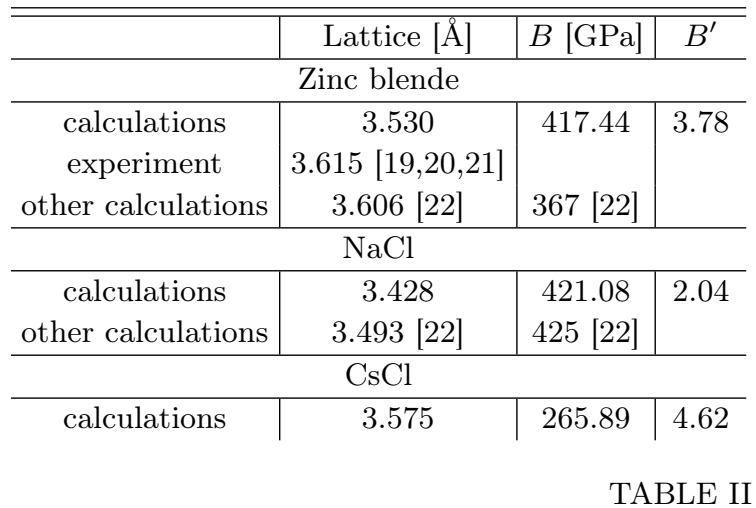

Structural properties of $\mathrm{BP}$ for zinc blende, $\mathrm{NaCl}$, and $\mathrm{CsCl}$.

\begin{tabular}{c|c|c|c}
\hline \hline & Lattice $[\AA]$ & $B[\mathrm{GPa}]$ & $B^{\prime}$ \\
\hline \multicolumn{4}{c}{ Zinc blende } \\
\hline calculations & 4.425 & 182.81 & 3.77 \\
experiment & $4.538[20,21]$ & & \\
other calculations & $4.558[22]$ & $166[22]$ & \\
\hline \multicolumn{4}{c}{$\mathrm{NaCl}$} \\
\hline calculations & 4.199 & 180.65 & 4.11 \\
other calculations & $4.339[22]$ & $155[22]$ & \\
\hline \multicolumn{4}{c}{$\mathrm{CsCl}$} \\
\hline calculations & 4.196 & 153.85 & 1.92
\end{tabular}

The band structure of $\mathrm{BN}, \mathrm{BP}$ and $\mathrm{BAs}$ for $\mathrm{ZB}$ are shown in Fig. 4, respectively. The results are given in Table IV and show that BN is a direct-gap semiconductor with the minimum of conduction band at $\Gamma, X$ point with energy gap $4.67 \mathrm{eV}$, and $\mathrm{BP}$ has an the indirect gap with minimum of conduction band at $\Gamma, \Delta_{\min }$ with energy gap $1.15 \mathrm{eV}$. The BAs has an indirect gap with minimum of conduction band at $\Gamma, \Delta_{\min }$ with energy gap $1.01 \mathrm{eV}$. There is a good agreement with experimental results. Valence charge density distribution in the zinc-blende structure at equilibrium volume for BN, BP, and BAs are given in Fig. 5.

TABLE III

Structural properties of BAs for zinc blende, $\mathrm{NaCl}$, and $\mathrm{CsCl}$.

\begin{tabular}{c|c|c|c}
\hline \hline & Lattice $[\AA]$ & $B[\mathrm{GPa}]$ & $B^{\prime}$ \\
\hline \multicolumn{4}{c}{ Zinc blende } \\
\hline calculations & 4.668 & 154.30 & 3.87 \\
experiment & $4.777[21]$ & & \\
other calculations & $4.777[22,23]$ & $145[22]$ & \\
\hline \multicolumn{4}{c}{$\mathrm{NaCl}$} \\
\hline calculations & 4.475 & 154.18 & 4.30 \\
other calculations & $4.583[22]$ & $143[22]$ & \\
\hline \multicolumn{4}{c}{$\mathrm{CsCl}$} \\
\hline calculations & 4.510 & 128.53 & 4.06
\end{tabular}



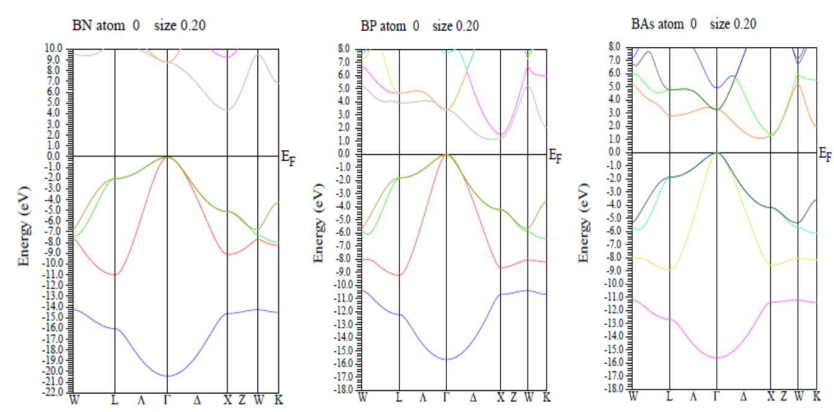

Fig. 4. Band-structures of zinc blende for the BN, BAs, and BP.

TABLE IV

Band gaps of BN, BP, and BAs in the zinc blende structure (all energies are in $\mathrm{eV}$ ).

\begin{tabular}{|c|c|c|}
\hline Compounds & Calculation & Other calculations \\
\hline \multicolumn{3}{|r|}{$\mathrm{BN}$} \\
\hline $\begin{array}{l}\text { direct gap } \\
\Gamma \longrightarrow x\end{array}$ & 4.67 & $\begin{array}{c}4.19[24], 4.40[25], 4.24[26], \\
4.6[27]\end{array}$ \\
\hline \multicolumn{3}{|r|}{$\mathrm{BP}$} \\
\hline $\begin{array}{l}\text { indirect gap } \\
\Gamma \longrightarrow \Delta_{\min } \\
\end{array}$ & 1.15 & $1.2[28], 1.9[29], 1.14[30]$ \\
\hline \multicolumn{3}{|c|}{ BAs } \\
\hline $\begin{array}{l}\text { indirect gap } \\
\Gamma \longrightarrow \Delta_{\min }\end{array}$ & 1.01 & $1.23[13], 1.60[30], 1.25[23]$ \\
\hline
\end{tabular}

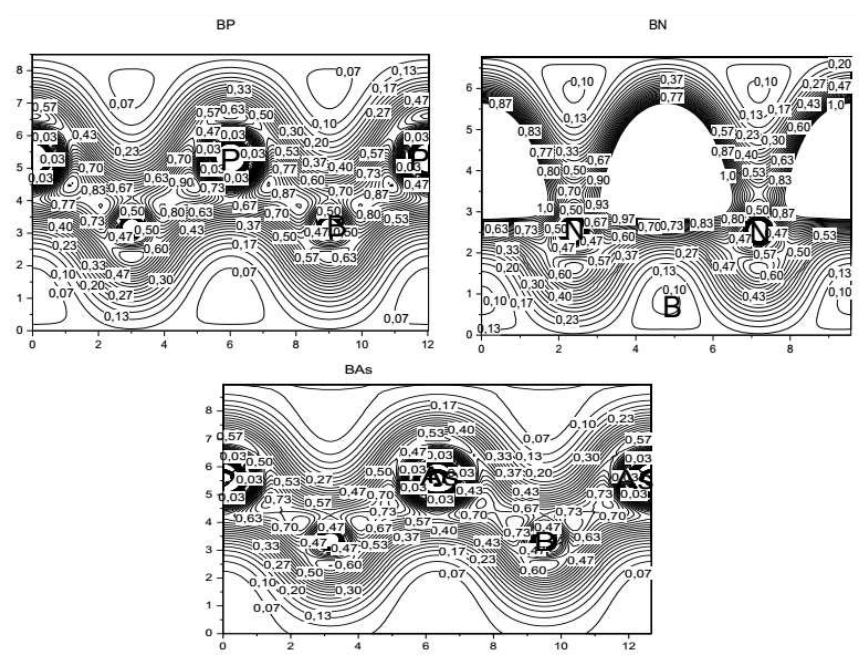

Fig. 5. Valence charge density distribution in the zinc blende structure at the equilibrium volume for $\mathrm{BN}, \mathrm{BP}$ and BAs.

The charge contours are shown in the (110) plane, if we show the last closed contour as an indication of the size of the atom, at the transition volume for BN. The N atoms are much larger than the $\mathrm{B}$ atoms but for $\mathrm{BP}$ and BAs. The $\mathrm{P}$ and As atoms are much smaller than the $\mathrm{B}$ atoms. The total density of states and partial BP (zinc blende) at equilibrium is given in Fig. 6. We can distinguish, from the origin of energy two important valence regions
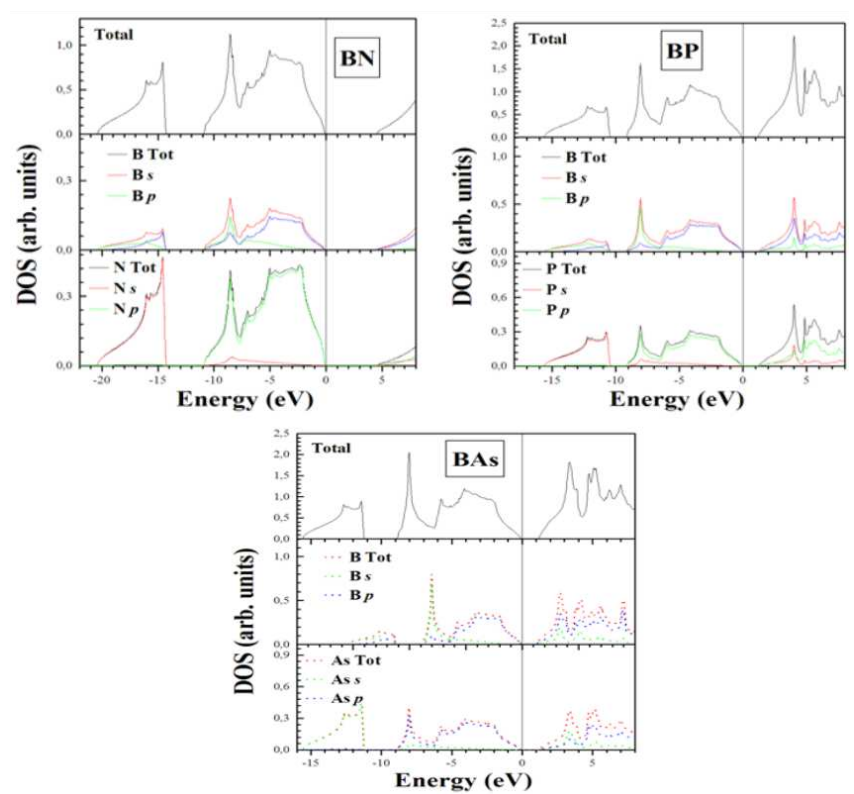

Fig. 6. States density distribution in the zinc blende structure at the equilibrium volume for $\mathrm{BN}, \mathrm{BP}$, and BAs.

called: VB1 and VB2. Hybridization is $s p$ type with a strong participation of the $p$ orbital in VB1 and $s$ orbital in the VB2. The $s$ orbital participation in the conduction band is less important than that of the $p$ orbital.

\section{Conclusion}

This work is a contribution to the study of structural and electronic properties of the materials based on boron and copper by the method FP-LAPW. The boronbased compounds BN, BP, and BAs crystallize in the zinc blende structure. We studied the relative stability of phases $\mathrm{NaCl}$ (rock salt) and $\mathrm{CsCl}$.

Our calculation of the electronic structure of BP and BAs indicates the presence of a fundamental indirect gap near $X\left(\Gamma \longrightarrow \Delta_{\min }\right)$, while for the BN we found a direct gap following $(\Gamma \longrightarrow x)$. These results agree with those of Wentzcovitch.

\section{References}

[1] J.A. Van Vechten, J.C. Phillips, Phys. Rev. B 2, 2160 (1970).

[2] A. Zaoui, M. Ferhat, B. Khelifa, J.P. Dufour, H. Aourag, Phys. Status Solidi B 185, 163 (1994).

[3] L. Kleinman, J.C. Phillips, Phys. Rev. 117, 460 (1960).

[4] P. Rodriguez-Hernandez, M. Gonzalez-Diaz, A. Munoz, Phys. Rev. B 51, 14705 (1995).

[5] L. Vel, G. Demazeau, J. Eloumeau, Mater. Sci. Eng. B 10, 149 (1991).

[6] E. Knittle, R.M. Wentzcovitch, R. Jeanloz, M.L. Cohen, Nature (London) 337, 349 (1989). 
[7] R. Wentzcovitch, K.J. Chang, M.L. Cohen, Phys. Rev. B 34, 1071 (1986).

[8] R. Wentzcovitch, M.L. Cohen, J. Phys. C 19, 6791 (1986).

[9] W. Sekkal, B. Bouhafs, H. Aourag, M. Certier, J. Phys.: Condens. Matter 10, 4975 (1998).

[10] M.P. Surh, S.G. Louie, M.L. Cohen, Phys. Rev. B 43, 9126 (1991).

[11] A. Rubio, J.L. Corkill, M.L. Cohen, E.L. Shirley, S.G. Louie, Phys. Rev. B 48, 11810 (1993).

[12] M. Ferhat, B. Bouhafs, A. Zaoui, H. Aourag, J. Phys. Condens. Matter 10, 7995 (1998).

[13] A. Zaoui, M. Ferhat, B. Khelifa, J.P. Dufour, H. Aourag, Phys. Stat. Sol. (b). 185, 163 (1994).

[14] P. Blaha, K. Schwarz, P. Sorantin, S.B. Trickey, Comput. Phys. Commun. 59, 399 (1990).

[15] J.P. Perdew, Y. Wang, Phys. Rev. B 45, 13244 (1992).

[16] P. Hohenberg, W. Kohn, Phys. Rev. 136, B864 (1964)

[17] W. Kohn, L.J. Sham, Phys. Rev. 140, A1133 (1965).

[18] A. Zaoui, F. El Haj Hassan, J. Phys. Condens. Matter 13, 253 (2001).

[19] E. Knittle, R.M. Wentzcovitch, R. Jeanloz, M.L. Cohen, Nature 337, 349 (1989).
[20] Landolt-Börnstein, New Series, Group 3, Vol. 17a, Eds. K.H. Hellwege, O. Madelung, 1983.

[21] S. Adachi, Properties of Group IV, III-V and II-VI Semiconductors, Department of Electronic Engineering, Gumma University, Japan 2005.

[22] R.M. Wentzcovitch, M.L. Cohen, P.K. Lam, Phys. Rev. B 36, 6058 (1987)

[23] R.M. Wentzcovitch, M.L. Cohen, J. Phys. C Solid State Phys. 19, 6791 (1986).

[24] H. Bross, R. Bader, Phys. Status Solidi B 191, 369 (1995).

[25] K.T. Park, K. Terakura, N. Hamada, J. Phys. C Solid State Phys. 20, 1241 (1987).

[26] A. Zaoui, M. Ferhat, Phys. Status Solidi B 225, 15 (2001).

[27] V.I. Gavrilenko, R.Q. Wu, Phys. Rev. B 61, 2632 (2000).

[28] H. Meradji, S. Drablia, S. Ghemid, H. Belkhir, B. Bouhafs, A. Tadjer, .

[29] B. Paulus, P. Fulde, H. Stoll, Phys. Rev. B 54, 2556 (1996).

[30] P. Rodriguez-Hernandez, M. Gonzalez-Diaz, A. Munoz, Phys. Rev. B 51, 14705 (1995). 